\title{
Clinical impact of albumin in advanced head and neck cancer patients with free flap reconstruction - a retrospective study
}

\author{
Ming-Hsien Tsai ${ }^{1}$, Hui-Ching Chuang ${ }^{1,2}$, Yu-Tsai Lin ${ }^{1,2}$, Hui Lu ${ }^{1}$, Wei-Chih Chen ${ }^{1,2}$, Fu-Min Fang ${ }^{2,3}$, \\ Chih-Yen Chien ${ }^{\text {Corresp. } 1,2}$ \\ ${ }^{1}$ Department of Otolaryngology, Kaohsiung Chang Gung Memorial Hospital and Chang Gung University College of Medicine, Kaohsiung, Taiwan \\ 2 Head and Neck Oncologic Group, Kaohsiung Chang Gung Memorial Hospital, Kaohsiung, Taiwan \\ 3 Department of Radiation Oncology, Kaohsiung Chang Gung Memorial Hospital and Chang Gung University College of Medicine, Kaohsiung, Taiwan \\ Corresponding Author: Chih-Yen Chien \\ Email address: cychien3965@adm.cgmh.org.tw
}

Background. Poor nutritional status among patients with advanced stage head and neck squamous cell carcinoma (HNSCC) is common. Albumin is a common indicator of nutritional status and has been shown to be a predictor of oncological outcomes and perioperative morbidity. This study aims to determine the prognostic value of the serum albumin level among patients with advanced HNSCC undergoing surgery with simultaneous free flap reconstruction. Methods. A total of 233 patients with advanced head and neck cancer undergoing tumor resection and immediate microvascular free flap reconstruction in a tertiary referral center were enrolled retrospectively between January 2009 and December 2011. Statistical analyses including Pearson'schi-squared test was used to determine whether there was a significant difference between each selected clinical factors and postoperative major wound infection. Multiple regression analysis was performed to reveal the relationship between postoperative major wound infection and clinical factors. Kaplan-Meier curves and multivariate Cox regression were applied to analyses survival outcome for overall survival (OS), disease-specific survival (DSS) and disease-free survival (DFS). Results. Postoperative serum albumin level $(p<0.001)$ and tumor location were both significantly associated with postoperative major wound infection $(p=0.018)$ in univariate analysis. Multiple regression analysis showed a higher risk of postoperative major wound infection among patients with postoperative hypoalbuminemia than in their counterparts (odds ratio [OR] 9.811, 95\% confidence interval [CI] [2.288, 42.065], $p=0.002$ ). Patients with a tumor located over the hypopharynx experienced increased risk of postoperative major wound infection (OR 2.591, 95\% CI [1.095, 6.129], $p$ $=0.030$ ). With respect to oncological outcomes, preoperative serum albumin level is a significant independent prognostic factor for overall survival (OS), disease-specific survival (DSS) and disease-free survival (DFS). Conclusions. Postoperative hypoalbuminemia is a useful indicator for the development of postoperative complications. In addition, 
preoperative hypoalbuminemia is a negative prognostic factor for patients who have undergone tumor excision and free flap reconstruction for the advanced stage of HNSCC. 
1 Title:

2 Clinical impact of albumin in advanced head and neck cancer patients with free flap

3 reconstruction - a retrospective study

4 Ming-Hsien Tsai, MD ${ }^{1}$, Hui-Ching Chuang, MD, PhD, FACS ${ }^{1,2,}$,

5 Yu-Tsai Lin, MD, FACS ${ }^{1,2}$, Hui Lu, MS ${ }^{1}$, Wei-Chih Chen, MD, FACS ${ }^{1,2}$,

6 Fu-Min Fang, MD, $\mathrm{PhD}^{2,3}$, Chih-Yen Chien, $\mathrm{MD}, \mathrm{FACS}^{1,2, *}$

$7 \quad{ }^{1}$ Department of Otolaryngology, Kaohsiung Chang Gung Memorial Hospital and Chang Gung

8 University College of Medicine, Kaohsiung, Taiwan

$9{ }^{2}$ Kaohsiung Chang Gung Head and Neck Oncology Group, Cancer Center, Kaohsiung Chang

10 Gung Memorial Hospital, Kaohsiung, Taiwan

$11{ }^{3}$ Department of Radiation Oncology, Kaohsiung Chang Gung Memorial Hospital and Chang

12 Gung University College of Medicine, Kaohsiung, Taiwan

$13 *$ Corresponding author: Chih-Yen Chien, MD, FACS

14 Department of Otolaryngology, Kaohsiung Chang Gung Memorial Hospital

15123 Ta-Pei Road, Niao Song District, Kaohsiung, 833, Taiwan

16 Tel: +886-7-7317123 ext. 2533; Fax: $+886-7-7313855$

17 E-mail address: cychien3965@adm.cgmh.org.tw 
19

\begin{abstract}
\end{abstract}
Background.

Poor nutritional status among patients with advanced stage head and neck squamous cell carcinoma (HNSCC) is common. Albumin is a common indicator of nutritional status and has been shown to be a predictor of oncological outcomes and perioperative morbidity. This study aims to determine the prognostic value of the serum albumin level among patients with advanced HNSCC undergoing surgery with simultaneous free flap reconstruction.

\title{
Methods.
}

A total of 233 patients with advanced head and neck cancer undergoing tumor resection and immediate microvascular free flap reconstruction in a tertiary referral center were enrolled retrospectively between January 2009 and December 2011. Statistical analyses including Pearson's chi-squared test was used to determine whether there was a significant difference between each selected clinical factors and postoperative major wound infection. Multiple regression analysis was performed to reveal the relationship between postoperative major wound infection and clinical factors. Kaplan-Meier curves and multivariate Cox regression were applied to analyses survival outcome for overall survival (OS), disease-specific survival (DSS) and disease-free survival (DFS).

\section{Results.}

Postoperative serum albumin level $(p<0.001)$ and tumor location were both significantly associated with postoperative major wound infection $(p=0.018)$ in univariate analysis. Multiple regression analysis showed a higher risk of postoperative major wound infection among patients with postoperative hypoalbuminemia than in their counterparts (odds ratio [OR] 9.811, 95\% confidence interval $[\mathrm{CI}][2.288,42.065], p=0.002)$. Patients with a tumor located over the hypopharynx experienced increased risk of postoperative major wound infection (OR 2.591, 95\% CI $[1.095,6.129], p=0.030)$. With respect to oncological outcomes, preoperative serum albumin 
43 level is a significant independent prognostic factor for overall survival (OS), disease-specific

44 survival (DSS) and disease-free survival (DFS).

45 Conclusions.

46 Postoperative hypoalbuminemia is a useful indicator for the development of postoperative

47 complications. In addition, preoperative hypoalbuminemia is a negative prognostic factor for

48 patients who have undergone tumor excision and free flap reconstruction for the advanced stage

49 of HNSCC. 
50

51

52

5

54

5

56

57

58

59

60

61

62

63

64

65

66

67

68

69

70

71

\section{Introduction}

1 The nutritional status of patients with head and neck squamous cell carcinoma (HNSCC) can affect the disease course and prognosis (Langius et al., 2013). Patients with HNSCC are vulnerable to malnutrition at the time of diagnosis and during treatment. It may be caused by the tumor itself or by treatment-related symptoms such as dysphagia, odynophagia, anorexia, mechanical obstruction and fatigue (Ravasco et al., 2003). Generally, poor nutrition status affects treatment compliance, quality of life and survival outcomes in patients with cancer (Ravasco et al., 2004). Serum albumin concentrations may be related to weight loss among patients with cancer due to changes in body mass and systemic inflammatory reactions (McMillan et al., 2001). Serum albumin has also been investigated as a predictor of cancer survival and perioperative outcomes in a variety of cancers. Only a few studies have focused on clinical impact on the level of serum albumin in head and neck cancer patients (Danan et al., 2016).

Surgical intervention with adequate free margins is important for disease control in patients with head and neck cancer; however, a large defect can lead to great morbidity, dysfunction and disfigurement (Chen et al., 2012). Microvascular free flap transfer for head and neck reconstructive surgery has become increasingly popular during the past 20 years (Dassonville et al., 2008). However, when microsurgical complications occur, they not only delay postoperative adjuvant therapy but also result in subsequent treatment failure (Jones et al., 2007). Currently, data are still limited regarding the role of hypoalbuminemia among patients with HNSCC who have undergone surgery for tumor removal with free flap reconstruction. The aim of this study is to determine the prognostic value of serum albumin level among patients with advanced HNSCC who have undergone surgery for HNSCC and free flap reconstruction. 


\section{Materials and Methods}

\section{Study population}

74 This study retrospectively enrolled patients who were diagnosed with HNSCC and

underwent surgical resection of tumor with microvascular free flap reconstruction simultaneously between January 2009 and December 2011 in Kaohsiung Chang Gung Memorial hospital, Taiwan. The inclusion criteria were age $>20$ years and advanced stage (stage III/ IV) of HNSCC. The patients underwent regular examinations of body weight and circulatory laboratory data before surgery. In total, 233 patients (220 men and 13 women) with a median age of 54 years (30-84 years) were included for analyses. Tumors were re-staged according to the eighth edition of the American Joint Committee on Cancer Staging (AJCC). Treatment was primarily based on the American National Comprehensive Cancer Network (NCCN) guidelines. Concurrent chemoradiation (CCRT) or radiation was conducted for these cases. The chemotherapy agent was cisplatin-based. The radiation technique for these patients was intensity-modulated radiation therapy (IMRT). The primary radiation dose for HNSCC was between 6,800 and 7,000 cGy for gross disease. The mean adjuvant radiation dose was 6,000 cGy.

\section{Variables and Outcomes}

Patients were retrospectively enrolled according to the following clinical characteristics: gender, age, primary tumor site, cancer stage and follow-up information about recurrence and survival after treatment. Body mass index $\left(\mathrm{BMI}, \mathrm{kg} / \mathrm{m}^{2}\right)$ and circulatory laboratory data including serum albumin were regularly measured within one week before the surgery. The definition of postoperative serum albumin is the serum albumin level collected the morning following the surgery. Hypoalbuminemia is defined as serum albumin level $<3.5 \mathrm{~g} / \mathrm{dL}$. Major postoperative wound infection is defined as a postoperative recipient-site wound condition that necessitated wound debridement in the operation room.

\section{Statistical analysis}


97 Statistical analyses were performed using IBM SPSS Version 20.0 software (SPSS/IBM,

98 Inc., Chicago, IL). The end points of this study included postoperative wound infection, 5-year

99 disease-free survival (DFS), 5-year disease-specific survival (DSS) and 5-year overall survival

100 (OS). Pearson's chi-squared test was used to determine whether there was a significant difference

101 between each selected clinical factors and postoperative major wound infection. Multiple

102 regression analysis was performed to reveal the relationship between postoperative major wound

103 infection and clinical factors. The Kaplan-Meier method was utilized to estimate survival, and the

104 log-rank test was used to examine statistical significance of factors. Cox proportional hazards

105 models were used to identify significant variables associated with survival. The estimated hazard

106 ratios (HRs) and 95\% confidence intervals (CIs) were calculated. A two-tailed $p$ value $<0.05$ was

107 considered significant. This study was approved by the Medical Ethics and Human Clinical Trial

108 Committees at Chang Gung Memorial Hospital (Ethical Application Reference number:

109 201701238B0). 
110

111

112

113

114

115

116

117

118

119

120

121

122

123

124

125

126

127

128

129

130

131

132

\section{Results}

A total of 233 patients were enrolled in this study. The clinical characteristics of the study patients are summarized in Table 1 . The median age of patients was 54 years (range: $30-84$ ). The population included $220(94.4 \%)$ male patients and $13(5.6 \%)$ female patients. The average BMI in this population was $23.03 \mathrm{~kg} / \mathrm{m}^{2}$ (range: 14.7-42.6). The average preoperative serum albumin level was $4.2 \mathrm{~g} / \mathrm{dL}$ (range: $2.5-5.8$ ). The average postoperative serum albumin level was $3.1 \mathrm{~g} / \mathrm{dL}$ (range: 1.9-4.3). In this cohort, 53 patients (22.7\%) had type II diabetes mellitus and 24 patients $(10.3 \%)$ had chronic renal disease. The sole histopathologic cancer type in this population was squamous cell carcinoma. The most common tumor subsite was the oral cavity $(\mathrm{n}=156,66.9 \%)$, followed by the oropharynx $(n=31,13.3 \%)$, hypopharynx $(n=27,11.6 \%)$ and larynx $(n=19$, $8.2 \%$ ), respectively. All oropharyngeal cancers in this cohort were p16 negative tumors. There were 59 patients who underwent salvage surgery for recurrent or persistent HNSCC, 49 of whom had had prior radiotherapy. The flap was most often harvested from the anterolateral thigh (ALT) $(n=229,98.3 \%)$, followed by the anteromedial thigh $($ AMT $)(n=3,1.3 \%)$ and radial forearm $(n$ $=1,0.4 \%)$. The incidence of postoperative major wound infection was 60 (25.8\%). There was no postoperative donor site wound infection or complication in the population. Patients were followed up for a median of 53.9 months in this cohort. Tumor recurrence occurred in $75(32.2 \%)$ patients, including local, regional and distant recurrence of $26(34.7 \%), 30(40.0 \%)$ and 19 (25.3\%) patients, respectively. At the time of the last follow-up, 154 (66.1\%) patients remained disease-free, $51(21.9 \%)$ patients had died of the disease and $28(12 \%)$ patients had died of other diseases. The 5-year OS rate in this cohort is $65.5 \%$.

We were interested in the effects of the clinical factors, especially the albumin level affecting the postoperative major wound infection. The chi-square test for associations between 
133 each clinical factor and postoperative major wound infection were applied univariately and the p-

134 values are listed in Table 2.

135 Several factors influencing postoperative wound infection were selected for univariate analysis

136 (Table 2). Postoperative lower serum albumin level $(p<0.001)$ and primary cancer site over

137 hypopharynx $(p=0.018)$ were both significantly associated with higher probability of

138 postoperative major wound infection. To avoid the collinearity of predictors in a regression

139 model, chi-square test of association between postoperative lower serum albumin level and

140 primary cancer site over hypopharynx was performed. The demonstration shown us there was no

141 statistically significant association between the two predicting variables (chi-square test statistic $=$

$1420.468, p=0.494)$. A multiple regression analysis then was applied to analyze the relationship

143 between postoperative major wound infection and the two uncorrelated and significant factors

144 revealed in prior univariate analyses. In this model, postoperative serum albumin level was a

145 significant independent predictor of major wound infection (OR 9.811, 95\% CI [2.288, 42.065],

$146 p=0.002)$. In addition, tumor location over the hypopharynx significantly increased the

147 probability of postoperative major wound infection as compared to other cancer sites (OR 2.591,

$14895 \%$ CI $[1.095,6.129], p=0.030)($ Table 3$)$.

149 In this study we also evaluated prognostic factors such as cancer stage, whether or not the

150 patient had undergone salvage surgery, cancer site, pre- or post-operative hypoalbuminemia, body

151 mass index (BMI) and diabetes mellitus that could have an impact on survival. Five-year survival

152 rates of DSS, DFS and OS were calculated for all factors and are listed in Table 4. These results

153 show that salvage surgery type ( $p$ value: OS: $<0.001$; DSS: $<0.001$; DFS: 0.004 ) and preoperative

154 hypoalbuminemia ( $p$ value: OS: 0.011; DSS: 0.02; DFS: 0.036) are significantly associated with

155 lower rates of survival of OS, DSS and DFS. Kaplan-Meier survival curves were drawn for two

156 preoperative albumin levels (Fig. 1). The effects of hypoalbuminemia on survival were evaluated 
157 by fitting all the variables whose $p$-value $<0.25$ in the Kaplan-Meier survival analyses to the Cox

158 Regression model. The final analyses including the hypoalbuminemia variable for different

159 survival outcomes were conducted, and the results are listed in Table 5. Only the preoperative

160 hypoalbuminemia prognostic factor remained in the Cox Regression modeling. The results

161 demonstrate that preoperative hypoalbuminemia was the independent prognostic factor of OS

162 (hazard ratio [HR] 2.005, 95\% CI [1.021, 3.936], $p=0.043)$, DSS (HR 2.503, 95\% CI [1.126,

$1635.563], p=0.024)$ and DFS (HR 2.101, 95\% CI [1.046, 4.211], $p=0.037)$. 
164 Discussion

165 Nowadays, microsurgical free flap transfer is the mainstay of reconstruction for large defects

166 in the head and neck regions after tumor excision (Ariyan et al., 1997; Lutz \& Wei, 2005).

167 Successful head and neck reconstruction in cancer patients is crucial because delayed wound

168 healing may adversely affect the oncologic outcomes of these patients and increase the length of

169 hospital stay and cost. Several studies have investigated the importance of serum albumin level in 170 postoperative wound infection among patients who have undergone head and neck cancer

171 resection and simultaneous free tissue transfer. Lo et al. revealed that low albumin level was

172 associated with a greater tendency to develop postoperative complications in 61 patients who

173 underwent head and neck cancer surgery and simultaneous free tissue transfer (Lo et al., 2017).

174 Danan et al. identified a significant correlation between preoperative hypoalbuminemia and

175 increased probability of wound infection among patients with head and neck cancer (Danan et al.,

176 2016). Another report also found that postoperative low albumin level is associated with

177 postoperative complications after free flap transfer (Hoppe et al., 2012). In agreement with these

178 studies, postoperative hypoalbuminemia carried a higher risk of postoperative major wound

179 infection in our series. Basically, a decrease in serum albumin is associated with a decrease in

180 important serum proteins of the immune system, which are essential for infection and tumor

181 control. Although preoperative serum albumin level didn't show significant association with

182 major wound infection by multivariate analysis in this cohort, the postoperative albumin level

183 remained the significant factor affecting wound infection. Routine measurement of postoperative

184 albumin levels and adequate supply of postoperative nutrition by tube feeding are advised to

185 avoid potential wound complication and to shorten the hospital stay.

186 Primary cancer location is another variable affecting the postoperative wound infection,

187 especially among patients of hypopharyngeal cancer undergoing total laryngopharyngectomy. 
188 The tubed free flap harvested from the anterior lateral thigh showed higher incidence of

189 pharyngocutaneous fistula (PCF). This is likely a result of inadequate nutrition status during

190 treatment, the complexity of the surgical procedure and early wound breakdown from the free

191 flap. This type of fistula may further jeopardize the pedicle of the free flap, causing the loss of the

192 free flap and even massive bleeding from the pedicle. The PCF may also intensify the

193 psychological stress of patients, lead to serious wound complications, prolong the hospital stay,

194 increase treatment costs and even hamper the patient's chance of survival.

The relationship between hypoalbuminemia and prognosis of treatment outcome among

patients with cancer was noted in some studies. One systemic review in 2010 reported that higher serum albumin levels in patients would lead to higher rates of survival of gastrointestinal cancer (Gupta \& Lis, 2010). There are also limited studies reporting the prognostic significance of pretreatment hypoalbuminemia in head and neck cancer patients. Advanced cancer stage is the strongest predictor of OS in patients with HNSCC. In patients with stage IV disease, advanced age and low serum albumin were associated with poorer OS (Medow et al., 2002). A recent study revealed that preoperative hypoalbuminemia would lead to a higher probability of postoperative wound infection and poorer OS (Danan et al., 2016). Another study showed that pretreatment albumin level was one of the independent predictors of DFS, cancer-specific survival and OS.

Patients with HNSCC showing hypoalbuminemia prior to treatment would experience mortality (Lim et al., 2017). Our study also revealed similar results, showing preoperative hypoalbuminemia to be associated with poorer oncologic outcomes. These findings will prompt the clinicians to be cautious about the clinical impact of hypoalbuminemia in the surgical treatment of HNSCC.

\section{Conclusions}


212 Postoperative hypoalbuminemia is useful as an indicator for the development of

213 postoperative complications. In addition, preoperative hypoalbuminemia is a negative prognostic

214 factor among patients who undergo tumor excision and free flap reconstruction for advanced

215 stage of HNSCC. 
216

217

218

219

220

221

222

223

224

225

226

227

228

229

230

231

232

233

234

235

236

237

238

239

240

241

242

243

244

245

246

247

248

249

250

251

252

253

254

255

256

257

\section{References}

Ariyan S, Ross DA, and Sasaki CT. 1997. Reconstruction of the head and neck. Surg Oncol Clin N Am 6:143.

Chen YW, Chen CY, Chiang SC, Lui MT, Kao SY, and Yang MH. 2012. Predictors and impact of microsurgical complications in patients with locally advanced oral squamous cell carcinoma. Cancer Sci 103:1672-1678. 10.1111/j.1349-7006.2012.02345.x

Danan D, Shonka DC, Jr., Selman Y, Chow Z, Smolkin ME, and Jameson MJ. 2016. Prognostic value of albumin in patients with head and neck cancer. Laryngoscope 126:1567-1571. 10.1002/lary.25877

Dassonville O, Poissonnet G, Chamorey E, Vallicioni J, Demard F, Santini J, Lecoq M, Converset S, Mahdyoun P, and Bozec A. 2008. Head and neck reconstruction with free flaps: a report on 213 cases. Eur Arch Otorhinolaryngol 265:85-95. 10.1007/s00405-007-0410-1

Gupta D, and Lis CG. 2010. Pretreatment serum albumin as a predictor of cancer survival: a systematic review of the epidemiological literature. Nutr J 9:69. 10.1186/1475-2891-9-69

Hoppe IC, Abernathie BL, and Datiashvili RO. 2012. Examination of possible predictors of complications after free tissue transfer to the head and neck for oncologic defects. Ann Plast Surg 69:368-370. 10.1097/SAP.0b013e318246e6b4

Jones NF, Jarrahy R, Song JI, Kaufman MR, and Markowitz B. 2007. Postoperative medical complications-not microsurgical complications--negatively influence the morbidity, mortality, and true costs after microsurgical reconstruction for head and neck cancer. Plast Reconstr Surg 119:2053-2060. 10.1097/01.prs.0000260591.82762.b5

Langius JA, Bakker S, Rietveld DH, Kruizenga HM, Langendijk JA, Weijs PJ, and Leemans CR. 2013. Critical weight loss is a major prognostic indicator for disease-specific survival in patients with head and neck cancer receiving radiotherapy. Br J Cancer 109:1093-1099. 10.1038/bjc.2013.458

Lim WS, Roh JL, Kim SB, Choi SH, Nam SY, and Kim SY. 2017. Pretreatment albumin level predicts survival in head and neck squamous cell carcinoma. Laryngoscope. 10.1002/lary.26691

Lo SL, Yen YH, Lee PJ, Liu CC, and Pu CM. 2017. Factors Influencing Postoperative Complications in Reconstructive Microsurgery for Head and Neck Cancer. J Oral Maxillofac Surg 75:867-873. 10.1016/j.joms.2016.09.025

Lutz BS, and Wei FC. 2005. Microsurgical workhorse flaps in head and neck reconstruction. Clin Plast Surg 32:421-430, vii. 10.1016/j.cps.2005.02.006

McMillan DC, Watson WS, O'Gorman P, Preston T, Scott HR, and McArdle CS. 2001. Albumin concentrations are primarily determined by the body cell mass and the systemic inflammatory response in cancer patients with weight loss. Nutr Cancer 39:210-213. 10.1207/S15327914nc392_8

Medow MA, Weed HG, and Schuller DE. 2002. Simple predictors of survival in head and neck squamous cell carcinoma. Arch Otolaryngol Head Neck Surg 128:1282-1286.

Ravasco P, Monteiro-Grillo I, Vidal PM, and Camilo ME. 2003. Nutritional deterioration in cancer: the role of disease and diet. Clin Oncol (R Coll Radiol) 15:443-450.

Ravasco P, Monteiro-Grillo I, Vidal PM, and Camilo ME. 2004. Cancer: disease and nutrition are key determinants of patients' quality of life. Support Care Cancer 12:246-252. 10.1007/s00520-0030568- 


\section{Table $\mathbf{1}$ (on next page)}

Clinicopathological characteristics of 233 patients with advanced head and neck cancer resection with free flap transfer 
Clinicopathological characteristics of 233 patients with advanced head and neck cancer resection with free flap transfer

\begin{tabular}{|c|c|c|c|}
\hline Characteristics & & Value & $\%$ \\
\hline Median Age (year) [range] & & $0,84]$ & \\
\hline \multirow[t]{2}{*}{ Sex } & male & 220 & 94.4 \\
\hline & female & 13 & 5.6 \\
\hline Average BMI $(\mathrm{Kg} / \mathrm{m} 2)$ [range] & & $4.7,42.6]$ & \\
\hline Average preoperative albumin (g/dL) [range] & & $.5,5.8]$ & \\
\hline Average postoperative albumin $(\mathrm{g} / \mathrm{dL})$ [range] & & $.9,4.3]$ & \\
\hline Diabetes mellitus & & 53 & 22.7 \\
\hline Chronic renal disease & & 24 & 10.3 \\
\hline Postoperative major wound infection & & 60 & 25.8 \\
\hline Cancer type & $\mathrm{SCC}$ & 233 & 100.0 \\
\hline \multirow[t]{2}{*}{ Cancer stage } & III & 45 & 19.3 \\
\hline & IV & 188 & 80.7 \\
\hline \multirow[t]{2}{*}{ Salvage surgery } & No & 174 & 74.7 \\
\hline & Yes & 59 & 25.3 \\
\hline \multirow[t]{2}{*}{ Preoperative radiotherapy } & No & 184 & 79.0 \\
\hline & Yes & 49 & 21.0 \\
\hline \multirow[t]{4}{*}{ Cancer site } & Oropharynx & 31 & 13.3 \\
\hline & Larynx & 19 & 8.2 \\
\hline & Hypopharynx & 27 & 11.6 \\
\hline & Oral Cavity & 156 & 66.9 \\
\hline \multirow[t]{3}{*}{ Flap design } & ALT & 229 & 98.3 \\
\hline & Radial forearm & 1 & 0.4 \\
\hline & AMT & 3 & 1.3 \\
\hline Median follow up time (months) [range] & & $.8,88.7]$ & \\
\hline \multirow[t]{2}{*}{ Recurrence } & Yes & 75 & 32.2 \\
\hline & No & 158 & 67.8 \\
\hline \multirow[t]{3}{*}{ Recurrent site } & Local & 26 & 34.7 \\
\hline & Regional & 30 & 40.0 \\
\hline & Distant & 19 & 25.3 \\
\hline \multirow[t]{3}{*}{ Last status } & NED & 154 & 66.1 \\
\hline & DOD & 51 & 21.9 \\
\hline & DWOD & 28 & 12 \\
\hline
\end{tabular}


BMI: body mass index

SCC: squamous cell carcinoma

ALT: anterolateral thigh

AMT: anteromedial thigh

NED: no evidence of disease

DOD: died of disease

DWOD: died without disease 


\section{Table 2 (on next page)}

Univariate analysis of risk factors impacting postoperative major wound infection of all patients 
Univariate analysis of risk factors impacting postoperative major wound infection of all patients

\begin{tabular}{llccc}
\hline Variable & & Number & $\begin{array}{c}\text { Major Wound } \\
\text { Infection }\end{array}$ & $p$ \\
\hline Stage & III & 45 & 9 & 0.326 \\
& IV & 188 & 51 & \\
Salvage surgery & No & 174 & 42 & 0.334 \\
& Yes & 59 & 18 & \\
Primary cancer site & Not hypopharynx & 207 & 48 & 0.018 \\
Preoperative serum & Hypopharynx & 26 & 12 & \\
albumin level (g/dL) & $<3.5$ & 16 & 6 & 0.253 \\
Postoperative serum & $\geqq 3.5$ & 217 & 54 & $<0.001$ \\
albumin level (g/dL) & $\geqq 3.5$ & 187 & 58 & \\
& $\geqq 3.5$ & 46 & 2 & 0.477 \\
Age & $<60$ & 160 & 39 & \\
& $\geqq 60$ & 73 & 21 & 0.826 \\
BMI (Kg/m2) & $<22$ & 96 & 24 & 0.817 \\
Diabetes Mellitus & $\geqq 22$ & 137 & 36 & \\
& No & 180 & 47 & 13 \\
\hline
\end{tabular}

1 


\section{Table 3(on next page)}

Regression analysis of factors impacting postoperative major wound infection of all patients 
Regression analysis of factors impacting postoperative major wound infection of all patients

\begin{tabular}{llcccc}
\hline Factor & & Odds ratio & $\begin{array}{c}95 \% \text { Confident } \\
\text { Interval }\end{array}$ & $p$ value \\
\hline \multirow{2}{*}{ Postoperative Albumin $(\mathrm{g} / \mathrm{dL})$} & $\geqq 3.5$ & 1 & & & .002 \\
& $<3.5$ & 9.811 & 2.288 & 42.065 & \\
Primary Cancer Site & Not Hypopharynx & 1 & & & .030 \\
& Hypopharynx & 2.591 & 1.095 & 6.129 & \\
\hline
\end{tabular}

1 


\section{Table 4(on next page)}

Univariate Analysis of risk factors impacting survival of all patients 
Univariate Analysis of risk factors impacting survival of all patients

\begin{tabular}{|c|c|c|c|c|c|c|c|c|c|c|c|}
\hline Variable & & Number & Event & OS (\%) & $p$ & Event & DSS (\%) & $p$ & Event & DFS (\%) & $p$ \\
\hline \multirow[t]{2}{*}{ Cancer stage } & III & 45 & 11 & 75.8 & 0.053 & 6 & 85.7 & 0.064 & 10 & 76.7 & 0.056 \\
\hline & IV & 188 & 69 & 61.6 & & 45 & 71.6 & & 65 & 62.7 & \\
\hline \multirow[t]{2}{*}{ Salvage surgery } & No & 174 & 49 & 71.3 & $<0.001$ & 29 & 81 & $<0.001$ & 48 & 70.7 & 0.004 \\
\hline & Yes & 59 & 31 & 44.8 & & 22 & 55.6 & & 27 & 50.2 & \\
\hline \multirow[t]{2}{*}{ Primary cancer site } & Not Hypopharynx & 206 & 70 & 65.3 & 0.881 & 43 & 75.8 & 0.648 & 65 & 66.1 & 0.908 \\
\hline & Hypopharynx & 27 & 10 & 56.6 & & 8 & 66.2 & & 10 & 61.8 & \\
\hline Preoperative albumin & $<3.5$ & 16 & 10 & 34.4 & 0.011 & 7 & 49.2 & 0.02 & 9 & 39.7 & 0.036 \\
\hline level (g/dL) & $\geqq 3.5$ & 217 & 70 & 66.9 & & 44 & 76.4 & & 66 & 67.4 & \\
\hline Postoperative albumin & $<3.5$ & 187 & 65 & 64.8 & 0.807 & 41 & 74.8 & 0.987 & 56 & 66.7 & 0.781 \\
\hline level (g/dL) & $\geqq 3.5$ & 46 & 15 & 62.6 & & 10 & 73.3 & & 16 & 61.8 & \\
\hline \multirow[t]{2}{*}{ BMI (Kg/m2) } & $<22$ & 96 & 35 & 61.8 & 0.532 & 24 & 70.8 & 0.353 & 32 & 64 & 0.824 \\
\hline & $\geqq 22$ & 137 & 45 & 66.3 & & 27 & 77.2 & & 43 & 66 & \\
\hline \multirow[t]{2}{*}{ Diabetes mellitus } & No & 180 & 59 & 66.8 & 0.451 & 39 & 74.9 & 0.897 & 60 & 64.6 & 0.523 \\
\hline & Yes & 53 & 21 & 56.7 & & 12 & 73.5 & & 15 & 68 & \\
\hline
\end{tabular}

\section{OS: overall survival}

DSS: disease specific survival

DFS: disease free survival 


\section{Table 5 (on next page)}

Cox Regression Analysis (proportional hazard analysis) of prognostic factors to survival of all patients 
Cox Regression Analysis (proportional hazard analysis) of prognostic factors to survival of all patients

\begin{tabular}{|c|c|c|c|c|c|}
\hline Factor & & Hazard ratio & \multicolumn{2}{|c|}{$\begin{array}{c}95 \% \text { Confident } \\
\text { Interval }\end{array}$} & $p$ value \\
\hline \multicolumn{6}{|c|}{ Proportional hazard analysis of overall survival (OS) } \\
\hline \multirow[t]{2}{*}{ Preoperative albumin level $(\mathrm{g} / \mathrm{dL})$} & $\geqq 3.5$ & 1 & & & \multirow[t]{2}{*}{.014} \\
\hline & $<3.5$ & 2.307 & 1.188 & 4.480 & \\
\hline \multicolumn{6}{|c|}{ Proportional hazard analysis of disease specific survival (DSS) } \\
\hline \multirow[t]{2}{*}{ Preoperative albumin level $(\mathrm{g} / \mathrm{dL})$} & $\geqq 3.5$ & 1 & & & \multirow[t]{2}{*}{.024} \\
\hline & $<3.5$ & 2.503 & 1.126 & 5.563 & \\
\hline \multicolumn{6}{|c|}{ Proportional hazard analysis of disease free survival (DFS) } \\
\hline \multirow[t]{2}{*}{ Preoperative albumin level $(\mathrm{g} / \mathrm{dL})$} & $\geqq 3.5$ & 1 & & & \multirow[t]{2}{*}{.037} \\
\hline & $<3.5$ & 2.101 & 1.046 & 4.211 & \\
\hline
\end{tabular}




\section{Figure 1 (on next page)}

\section{Kaplan-Meier survival curves}

Kaplan-Meier survival curves of overall survival (A), disease-specific survival (B), and diseasefree survival $(C)$ according to the serum albumin level before operation 


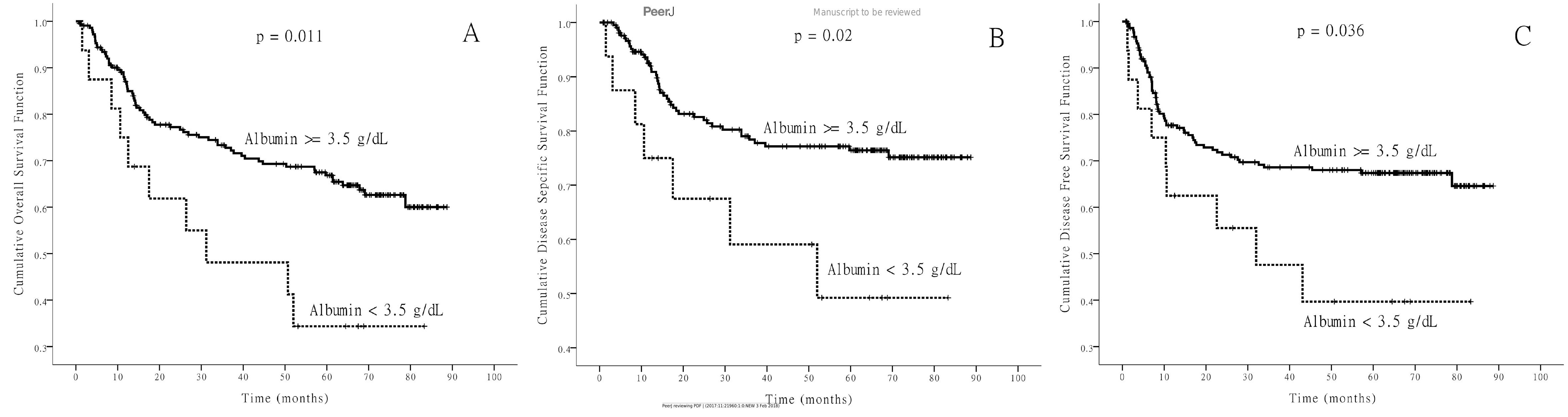

